Methods We have compared the Inspiratory Flow rate (I) with the flow metre against Maximum Inspiratory flows taken from a flow volume loop (FVI) taken as part of routine lung function testing in 100 sequential subjects attending the Cardio Respiratory department for lung function testing.

Results We have found major variability in the FVI on flow volume traces despite attempts to obtain traces with maximum volume and effort. Only $36 \%$ of subjects had variability between attempts of $<1 \mathrm{l} /$ s, with $64 \%$ showing variability between attempts of $>11 / \mathrm{s}, 24 \%$ of $>21 / s$, and $3 \%>31 / s$. For measurements using the Inspiratory Flow metre If I $>21 / \mathrm{s}$ all of the $38 \%$ of subjects showed FVI of $21 / \mathrm{s}$ or more. With I of $<21 / \mathrm{s}$ there was agreement between the two methods $\pm 0.3 \mathrm{l} / \mathrm{s}$ in $26 \%$, and a further $14 \%$ with FVI of $<2 \mathrm{l} / \mathrm{s}$. $40 \%$ of subjects with Inspiratory Flow (I) of $<21 / \mathrm{s}$ had FVI of $<21 / \mathrm{s}$. But in $22 \%$ of subjects I $<21 / \mathrm{s}$ but FVI $>21 / \mathrm{s}$. FVI-I showed mean difference for these subjects of $2.4 \mathrm{l} / \mathrm{s}$ (range $0.9-4 \mathrm{l} / \mathrm{s}$ ). In total $78 \%$ of subjects showed concordance of Maximum Inspiratory Flow to $>21 / \mathrm{s}$ or $>2 \mathrm{l} /$ $s$ between the two measurements and for $22 \%$ the inspiratory flow metre reading of $<2 \mathrm{l} / \mathrm{s}$ did not reflect maximum Inspiratory Flow.

Conclusion There are major variations in the Maximum Inspiratory Flow measured with a flow volume loop but for a simpler measurement with an Inspiratory Flow metre if Maximum flow is $>21 /$ min then it is unlikely that Inspiratory flow is compromised. A simple clinic based measurement can be useful to exclude limitation of Inspiratory Flow but if abnormal further investigation is needed.

\section{P136 COMPARISON BETWEEN PRIMARY CARE AND SECONDARY CARE SPIROMETRY}

doi:10.1136/thoraxjnl-2011-201054c.136

K E Backler, L Leech, R C Buttery. Hinchingbrooke Hospital NHS Trust, Huntingdon, UK

Introduction Spirometric testing in primary care is promoted by the QoF for GPs. The validity of such tests is questionable, due to numerous factors, including poor technique, machine maintenance and interpretive skills. The COPD Strategy supports the use of quality-assured spirometry in primary care. This study assesses the accuracy of primary care-based spirometry in referrals to our chest clinic and new Direct Access Pulmonary Function service.

Aims

1. To validate GP spirometry values with Secondary care values.

2. To identify differences in diagnosis based on physiological measurements.

3. To identify changes in severity status on COPD patients.

Method An audit was conducted, comparing Spirometry performed in Primary care (various machines and various technicians) with Spirometry performed on the Masterscreen PFT (CareFusion) in Lung Function laboratory. Where appropriate, obstructive spirometry was classified using GOLD/NICE COPD guidelines.

Results 37 patients identified.

No Spirometry results from GP $=4(11 \%)$

No change $=17(46 \%)$

Changed $=16(43 \%)$

Of the 16 that had their diagnosis changed:

- $5(31 \%)$ classified as restrictive on referral, but were normal

- $4(25 \%)$ classified obstructive on referral, but were normal

- 7 (44\%) classified as normal on referral, but were obstructive Of all referrals which were classified as obstructive (22 patients), $64 \%$ had their GOLD severity changed:

- 8 maintained their severity as classified by GP spirometry (36\%)

- 8 changed by 1 GOLD stage $(36 \%)$

- 6 changed 2 GOLD stages (27\%)

Conclusion For patients with COPD, the cost in treating patients varies with their disease severity. A change in severity staging would significantly alter the cost of treatment for Primary Care, by influencing the appropriate choice of treatment interventions. Correct diagnosis in primary care is fundamental to appropriate treatment and referral pathways for patients with respiratory disease. This study identifies a significant difference in physiological diagnosis achieved in secondary care and supports the need for more qualityassured pulmonary function testing.

\section{P137 INTERPRETATION OF PLETHYSMOGRAPHY IN HEALTHY YOUNG CHILDREN}

doi:10.1136/thoraxjnl-2011-201054c.137

${ }^{1} \mathrm{~J} C$ Kirkby, ${ }^{1} \mathrm{R}$ Bonner, ${ }^{1} \mathrm{~S}$ Lum, ${ }^{2} \mathrm{P}$ Bates, ${ }^{2} \mathrm{~V}$ Morgan, ${ }^{2} \mathrm{R}$ Strunk, ${ }^{1} \mathrm{~F}$ Kirkham, ${ }^{1} \mathrm{~S}$ Sonnappa, ${ }^{1} \mathrm{~J}$ Stocks. ${ }^{1}$ UCL, Institute of Child Health, London, UK; ${ }^{2}$ Washington University, Missouri, USA

Introduction Plethysmographic lung volumes are the gold standard for identifying restrictive lung defects (reduced TLC), and are useful for delineating obstructive defects (increased RV/TLC). ${ }^{1}$ Interpretation of these measurements may, however, be limited without appropriate reference equations. The BTS recommend equations by Rosenthal $^{2}$ (based on white subjects) for children. However, to our knowledge, no ethnic-specific plethysmographic equations have been published for black children, in whom lower spirometric values are known to exist.

Aim To evaluate the appropriateness of plethysmographic reference equations in healthy young children according to ethnic origin.

Methods Healthy children (68 black and 115 white) aged 6-12 yrs underwent plethysmography measurements according to standardised guidelines. ${ }^{1}$ Results were adjusted for sex and height and expressed as \%predicted and z-scores using recommended equations. ${ }^{2}$ Unpaired t-tests were used to establish ethnic differences.

Results Ethnic differences in lung volumes were dependent on the outcome: Black children had significantly lower FRC ( $\sim 6 \%$ or $0.3 z)$ and TLC ( $\sim 8 \%$ or $0.6 z)$, but no significant differences in RV such that their RV/TLC ratio was significantly higher (Abstract P137 table 1). In addition, relatively poor agreement between observed vs predicted FRC was seen in healthy white children. To avoid misdiagnosis, the limits of normality (mean $\pm 2 \mathrm{SD}$ ) need to be adjusted to cater for these discrepancies. These preliminary data suggest that, based on the Rosenthal equations, the lower limit of normal for TLC, (to detect restriction), would be $\sim 75 \%$ predicted $(-2.1 \mathrm{z}$ ) for black children and $\sim 80 \%$ predicted $(-1.7 z)$ for white children. For detecting hyperinflation using RV/TLC the upper limit of normal would be $\sim 148 \%$ predicted (2.3z) for black children and $\sim 135 \%$ predicted $(1.7 z)$ in white children, whereas for FRC they would be $\sim 111 \%$ predicted $(0.4 z)$ and $122 \%$ predicted $(1.2 z)$ in black and white children respectively.

Abstract P137 Table 1 Comparison of plethysmographic outcomes between 68 healthy black and 115 healthy white children

\begin{tabular}{lccc}
\hline & Black mean (SD) & White mean (SD) & $\begin{array}{l}\text { Mean diff (95\% CI) } \\
\text { (black-white) }\end{array}$ \\
\hline N (\% male) & $68(46 \%)$ & $115(45 \%)$ & \\
Age (yr) & $10.0(1.5)$ & $8.9(1.7)$ & $1.1(0.6 \text { to } 1.5)^{* *}$ \\
FRC \% pred & $86.2(12.6)$ & $91.2(15.4)$ & $-5.8(-10.1 \text { to }-1.4)^{*}$ \\
$\quad$ FRC z-score & $-0.7(0.6)$ & $-0.4(0.8)$ & $-0.3(-0.5 \text { to }-0.1)^{*}$ \\
RV \% pred & $103.7(20.0)$ & $99.0(23.4)$ & $4.7(-2.0$ to 11.4$)$ \\
RV z-score & $0.1(0.7)$ & $0(0.8)$ & $0.2(-0.1$ to 0.4$)$ \\
TLC \% pred & $94.2(9.8)$ & $101.7(11.0)$ & $-7.5(-10.6 \text { to }-4.3)^{* *}$ \\
TLC z-score & $-0.5(0.8)$ & $0.1(0.9)$ & $-0.6(-0.9 \text { to }-0.4)^{* *}$ \\
RV/TLC \% pred & $110.2(19.0)$ & $96.9(19.0)$ & $13.2(7.5 \text { to } 19.0)^{* *}$ \\
RV/TLC z-score & $0.5(0.9)$ & $-0.1(0.9)$ & $0.6(0.3 \text { to } 0.9)^{* *}$ \\
\hline
\end{tabular}

${ }^{*} p<0.05,{ }^{* *} p<0.0005$. 
Conclusion Ethnic differences in lung volumes exist and cannot be accounted for by a simple correction factor. Current paediatric plethysmographic reference equations are not appropriate, and may lead to misdiagnosis unless limits of normality are adjusted. Caution in interpretation is recommended until more appropriate reference equations can be developed. This requires prospective plethysmographic lung volume data collection using standardised protocols in children from different ethnic backgrounds.

\section{REFERENCES}

1. Wanger ERJ. 2005

2. Rosenthal. Thorax 1993.

\section{P138 EVALUATION OF THE NEED FOR A COMPREHENSIVE ASSESSMENT OF LUNG FUNCTION PRE OPERATIVELY IN THE MORBIDLY OBESE}

doi:10.1136/thoraxjnl-2011-201054c.138

A Renton, D A Lonsdale, G V Miller, J S White. York Teaching Hospital NHS Foundation Trust, York, UK

Introduction and Objectives Chronic morbid obesity has been shown to impinge on normal respiratory function and can ultimately result in respiratory failure. Lee et al (2010) concluded that a more physiological assessment of individuals in this group is required when they are investigated clinically. Accordingly, the clinical pathway of patients referred for bariatric surgery at York Hospital was changed to include comprehensive Pulmonary Function Tests (PFT's), as well as the current standard Overnight Pulse Oximetry. The objective was to evaluate the revised clinical service and distinguish if the additional measurements are appropriate for morbidly obese patients as part of their surgical pre-assessment.

Methods Data were collected from patients undergoing bariatric presurgical assessment, between January 2011 and June 2011. PFT's undertaken included dynamic lung volumes, static lung volumes and transfer factor for carbon monoxide ( $\mathrm{TL}_{\mathrm{CO}}$ ), using a Medgraphics Plethysmograph system in accordance with recognised standards. Overnight oximetry was performed using Minolta 300i pulse oximeters. Abnormalities of lung function were determined using the BTS (1997) guidelines. Obstructive sleep apnoea (OSA) was diagnosed using the Scottish Intercollegiate Guidelines Network, (2003) guidelines.

Results A total of 59 patients were included in the study with demographics and measurements summarised in Abstract P138 table $1.28 .8 \%$ had normal lung function and no evidence of OSA $39 \%$ had evidence of OSA but normal lung function. $6.8 \%$ had abnormal lung function but no OSA and $25.4 \%$ had both abnormal lung function and evidence of OSA.

Conclusions $\mathrm{Va}$ and $\mathrm{FRC}_{\text {Pleth }}$ are reduced in all groups but is more significant in both groups with abnormal lung function. Those with combined abnormalities are on average 14.8 and 6 years older than those with no abnormalities or just OSA respectively suggesting the development of these co-morbidities with age. We feel that the finding that $32.2 \%$ of bariatric referrals were found to have abnormal lung function justifies the inclusion of these measurements when assessing this category of patient. Also, further assessment following weight loss in order to establish the underlying nature of deficient lung function would be useful.

\section{P139 THE USE OF VENTURI MASKS WITH OXYGEN CONCENTRATORS}

doi:10.1136/thoraxjnl-2011-201054c.139

J C T Pepperell, C Fraser, G McClue. Taunton and Smerset NHS Foundation Trust, Taunton, UK

Background Venturi masks are commonly used in the hospital setting to deliver controlled percentages of oxygen. This is important for those at risk of hypercapnic respiratory failure and to accurately assess ventilation and gas exchange. Hospital oxygen supplies deliver high velocity and pressure, which are required for Venturi masks to operate as designed. Home oxygen is commonly delivered via concentrators, which work at lower pressures and this may affect the performance of Venturi masks if they are requested for home use.

Aim We aimed to review the oxygen delivered by the concentrator and the performance of Venturi barrels (24\%, 28\% 31\% and 35\%) with the Intensity concentrator at the usual flow rates used.

Methods Venturi barrels from the three manufacturers were tested using a TSI ${ }^{\circledR}$ Certifier ${ }^{\circledR}$ FA Test system, pressure gauge, flow metre and oxygen sensor. The Intensity concentrator was set at the specified flow rate and allowed to stabilise, oxygen concentration and outlet pressure were noted. We attached the Venturi barrels and recorded the flow rate, back pressure and delivered oxygen concentration. If the Venturi under performed we adjusted the concentrator to deliver the stated $\mathrm{FiO} 2$ and noted the flow rate required.

Results The concentrator delivered between $95.8 \%$ and $84.7 \%$ oxygen, with concentration reducing at higher flows (see Abstract

Abstract P138 table 1 Summary of demographic and respiratory data for pre bariatric surgery patients

\begin{tabular}{|c|c|c|c|c|c|}
\hline & $\begin{array}{l}\text { All subjects } \\
n=59\end{array}$ & $\begin{array}{l}\text { Abnormal PFT's } \\
\mathrm{n}=4\end{array}$ & $\begin{array}{l}\text { OSA } \\
n=23\end{array}$ & $\begin{array}{l}\text { Abnormal PFT's \& OSA } \\
n=15\end{array}$ & $\begin{array}{l}\text { No abnormalities } \\
n=17\end{array}$ \\
\hline Males & $\mathrm{n}=21$ & $n=2$ & $\mathrm{n}=9$ & $\mathrm{n}=7$ & $\mathrm{n}=3$ \\
\hline Females & $\mathrm{n}=38$ & $\mathrm{n}=2$ & $\mathrm{n}=14$ & $n=8$ & $\mathrm{n}=14$ \\
\hline Age (yrs) & $44.5 \pm 11.37$ & $38.8 \pm 16.76$ & $46.0 \pm 6.39$ & $52 \pm 9.79$ & $37.2 \pm 12.44$ \\
\hline BMI $\left(\mathrm{Kg} / \mathrm{m}^{2}\right)$ & $48.2 \pm 7.40$ & $43.3 \pm 5.56$ & $48.6 \pm 7.79$ & $47.2 \pm 8.61$ & $49.9 \pm 5.90$ \\
\hline $\mathrm{SpO}_{2}(\%)$ & $96.7 \pm 3.36$ & $97.8 \pm 1.50$ & $96.3 \pm 4.52$ & $95.8 \pm 3.10$ & $97.6 \pm 1.32$ \\
\hline Spirometry & Normal $=47$ & Normal $=3$ & Normal $=23$ & Normal $=5$ & Normal $=17$ \\
\hline \multirow[t]{2}{*}{ Pattern } & Obstructive $=4$ & Obstructive $=0$ & Obstructive $=0$ & Obstructive $=3$ & Obstructive $=0$ \\
\hline & Restrictive $=8$ & Restrictive $=1$ & Restrictive $=0$ & Restrictive $=7$ & Restrictive $=0$ \\
\hline FVC (\%pred) & $96.05 \pm 16.27$ & $86.25 \pm 4.76$ & $103.83 \pm 10.89$ & $75.80 \pm 7.88$ & $105.71 \pm 11.94$ \\
\hline $\mathrm{FRC}_{\text {pleth }}(\%$ pred$)$ & $76.50 \pm 16.76$ & $67.00 \pm 22.73$ & $82.14 \pm 15.37$ & $75.85 \pm 22.24$ & $71.94 \pm 10.80$ \\
\hline $\mathrm{TL}_{\mathrm{co}}(\%$ pred $)$ & $100.34 \pm 14.49$ & $89.50 \pm 17.33$ & $104.65 \pm 13.76$ & $94.33 \pm 15.00$ & $102.35 \pm 12.37$ \\
\hline $\mathrm{V}_{\mathrm{A}}(\%$ pred $)$ & $89.00 \pm 13.45$ & $78.75 \pm 5.12$ & $94.61 \pm 10.95$ & $75.40 \pm 10.08$ & $95.82 \pm 10.14$ \\
\hline $\mathrm{K}_{\mathrm{co}}(\% \mathrm{pred})$ & $113.75 \pm 18.94$ & $112.50 \pm 20.42$ & $110.83 \pm 13.74$ & $126.07 \pm 22.77$ & $107.12 \pm 17.53$ \\
\hline
\end{tabular}

Data presented as $\mathrm{n}$ or mean $\pm \mathrm{SD}$.

PFT's, pulmonary function tests; OSA, obstructive sleep apnoea; $\mathrm{BMI}$, body mass index; $\mathrm{SpO}_{2}$, saturation of peripheral oxygen; $\mathrm{FVC}$, forced vital capacity; \% pred, percent predicted; FRC ${ }_{\text {pleth }}$ functional residual capacity measured by plethysmography; $\mathrm{TL}_{\mathrm{CO}}$, Transfer factor of the lung for carbon monoxide; $\mathrm{V}_{\mathrm{A}}$, alveolar volume; $\mathrm{K}_{\mathrm{CO}}$, Transfer coefficient of carbon monoxide. 